\title{
L'auteur qui écrit pour les enfants peut-il échapper à la double autorité de l'auteur devant le public et de l'adulte devant l'enfant?
}

Can the Playwright Who Writes for Young People Avoid the Situation of Being a

Superior Authority above Both Audiences as a Writer and Children as an Adult?

Suzanne Lebeau

\section{(2) OpenEdition}

Journals

Édition électronique

URL : https://journals.openedition.org/recherchestravaux/777

DOI : 10.4000/recherchestravaux.777

ISSN : 1969-6434

Éditeur

UGA Éditions/Université Grenoble Alpes

Édition imprimée

Date de publication : 1 décembre 2015

Pagination : 51-60

ISBN : 978-2-84310-314-8

ISSN : 0151-1874

\section{Référence électronique}

Suzanne Lebeau, «L'auteur qui écrit pour les enfants peut-il échapper à la double autorité de l'auteur devant le public et de l'adulte devant l'enfant? », Recherches \& Travaux [En ligne], 87 | 2015, mis en ligne le 01 janvier 2017, consulté le 29 octobre 2021. URL : http://journals.openedition.org/ recherchestravaux/777; DOI : https://doi.org/10.4000/recherchestravaux.777 
Suzanne LebeAu

Auteure, compagnie Le Carrousel, Le Cube (Montréal)

\section{L'auteur qui écrit pour les enfants peut-il échapper à la double autorité de l'auteur devant le public et de l'adulte devant l'enfant?}

L'enfance, à travers l'histoire, les civilisations et les modes, a toujours été considérée comme un état transitoire, et l'enfant considéré comme un être humain en devenir, absent des discours philosophiques, des théories qui racontent l'homme et ses quêtes, l'homme et ses conquêtes. L'infans est celui qui ne parle pas. Le passage à celui qui n'a pas droit de parole s'est fait rapidement. L'enfance est une intense période d'apprentissages, de la formation du soi, de la construction de l'identité... Quand il est question d'enfant, on ne dit pas identité mais développement. Pourtant, l'enfance n'est jamais vécue comme un passage par les enfants qui sont dans le présent absolu et déterminant. Ils ne sont pas en attente de... Ils vivent, enracinés dans un «ici et maintenant» avec une étonnante capacité à ne pas hiérarchiser leurs occupations et à s'absorber totalement dans ce qu'ils font. Pourquoi donc l'enfant qui vit au présent est-il toujours traité comme un être "en devenir»?

Cette dichotomie entre le regard que les adultes portent sur l'enfance et ce que nous apprennent les enfants d'eux-mêmes est troublante. L'être humain se construit dans et par le regard de l'autre. "Le soi est créé par son appréhension d'un autre», dit Thomas McEvilley dans Le paradoxe de la romance ${ }^{\mathrm{I}}$. Nous existons et nous nous définissons dans et par le regard de l'autre, inévitable présence qui remet en question l'identité. C'est d'autant plus vrai pour

I. T. McEvilley, The Romance: A Paradox, 1987, p. I25. 
l'enfant. Donald Winnicott affirme que la construction identitaire est liée aux soins que l'enfant reçoit dans sa petite enfance et qui permettent l'ancrage de l'image de soi sur la transformation corporelle, l'investissement narcissique, et la construction d'un «idéal du moi » dans la relation aux autres à travers les attentes des parents. Or, cette relation de dépendance se transforme rapidement en relation d'autorité qu'il faut questionner et à laquelle il faut inventer des alternatives pour permettre la rencontre artistique.

L'enfance et l'enfant ne sont jamais sujets. Ils sont objets. Objet de soins, d'étude de la psychologie cognitive et de la pédagogie, sciences humaines réservées à l'étude de l'enfance. Comme le disait Bernhard Schlink dans Le liseur: "[...] la philosophie ne se soucie pas des enfants. Elle les a abandonnés à la pédagogie, qui s'en occupe bien mal ${ }^{2} »$. Bernhard Schlink n'a pas complètement tort. La pédagogie s'est presque uniquement consacrée à moduler les règles qui encadrent la relation de soumission didactique où l'enfant est celui qui apprend et l'adulte celui qui sait. Il y a eu des exceptions : Freinet en France a basé sa méthode d'enseignement sur la libre expression de l'enfant et le travail coopératif; Montessori, en Italie, féministe avant l'heure, a milité pour un vrai respect de l'enfant comme personne humaine; Korczak en Pologne, initiateur d'une révolutionnaire autogestion pédagogique, donnait aux enfants un réel pouvoir d'expression, de participation, d'association, de décision dans des conseils démocratiques, Pestalozzi, Summerhill, Decroly... Aucun de ces penseurs n’a réussi à ébranler de manière durable et définitive la relation de soumission didactique où l'adulte est celui qui sait et l'enfant celui qui doit apprendre.

\section{La rencontre artistique}

Comment la relation d'autorité entre adulte et enfant peut-elle dénaturer la rencontre artistique? L'auteur est en position d'autorité face au public. Il parle d'un point de vue sur le monde qui est le sien et le public a choisi... S'il est heurté ou bouleversé, il a le droit de crier, partir, créer un scandale, certains s'inscrivant dans l'Histoire. Provoqués par les spectateurs qui répondent à l'auteur, ces scandales donnent au théâtre sa vitalité. La situation de l'enfant est autre : il ne choisit pas ce qu'il va voir, n'a pas le droit de sortir, pas droit au scandale. La relation de l'auteur au spectateur est celle de l'adulte à l'enfant : une relation de bonne conduite et de soumission didactique qui déséquilibre et menace la rencontre de deux intimités, fondement de la rencontre artistique.

2. B. Schlink, Le liseur, Paris, Gallimard, coll. «Folio», I999, p. I60. 
L'auteur qui écrit pour le jeune public se retrouve dans une double position d'autorité : celle de l'artiste qu'on écoute et de l'adulte dont on connaît les droits et les pouvoirs.

Comment l'auteur qui choisit le jeune public peut-il assumer les obligations morales, éthiques, esthétiques qu'il se donne à lui-même et celles que voudrait lui imposer une société multiforme aux enjeux éducatifs éclatés qui ne devraient pas le concerner?

Comment l'auteur qui choisit le jeune public peut-il rejoindre des spectateurs qui sont essentiellement, au sens philosophique du terme, dans une relation inégalitaire?

\section{La rencontre avec le jeune public}

Ces questions se sont posées très tôt dans ma relation au jeune public que j'ai rencontré à vingt et un ans comme comédienne dans une Commedia Dell'arte. Je jouais une Colombine et le Pierrot était muet. Après chaque représentation, les enfants me parlaient normalement et ceux qui allaient vers le Pierrot lui parlaient par gestes... Pourquoi les enfants me parlaient-ils normalement et s'adressaient-ils au Pierrot suivant les conventions du spectacle? Je me suis d'abord demandé où est la frontière entre la réalité et la fiction. Puis, s'il y a un langage particulier pour toucher les jeunes spectateurs, quel serait ce langage? Les questions se multipliaient, faisant de l'écriture pour jeune public un territoire aussi sauvage par l'étendue à défricher, que policé par les règles du convenable imposées de l'extérieur.

En 1975 , j'écris un premier texte Ti-Jean voudrait ben s'marier, mais... et fonde Le Carrousel avec Gervais Gaudreault pour monter ce texte. Je ne veux pas m'étendre sur ces cinq premières années d'écriture que j'appelle d'innocence, mais je ne peux pas non plus l'évacuer complètement car, dans la participation qu'on expérimentait, il y avait déjà une ouverture aux enfants et le désir de transgresser les règles données, comme le dit Nicolas Faure :

Ti-Jean voudrait ben smarier, mais... suppose une relation pédagogique entre l'auteur et l'enfant, mais pas si inégalitaire que cela. Certes, il y a autre chose qu'une simple expression artistique, la volonté de se poser autant en éducateur qu'en créateur. Mais en assumant cette part du pédagogique, par le risque de l'improvisation, l'auteur trouve finalement une nouvelle forme d'égalité dans la relation. L'adulte n'est pas donneur de leçon (le sens n'est pas univoque), il est force de proposition ${ }^{3}$.

3. N. Faure, Le théâtre jeune public. Un nouveau répertoire, Rennes, Presses universitaires de Rennes, coll. «Le spectaculaire théâtre», 2009, p. 92. 
De 1975 à 1980 , j'écrivis cinq textes conçus selon un principe de participation active des enfants qui leur donnait un pouvoir non négligeable sur le déroulement dramatique. Les spectacles étaient bien reçus par les enfants et par les adultes. J'écrivais d'un point de vue d'énonciation non contestable de l'adulte qui sait et veut partager un savoir avec les enfants.

\section{Intuition d'une nouvelle posture d'écriture : Une lune entre deux maisons}

En 1976, mon fils naît. Il a à peine trois ans et je l'emmène au théâtre. Il voit tout, même si aucun spectacle n'a été pensé pour les petits, nombreux dans les salles. Je les observe écouter, perdre l'intérêt, revenir au spectacle, se passionner, attentive aux stimuli qui vont chercher leur attention, aux moments où ils décrochent. D'autre part, je travaille quatre jours par semaine en atelier de création libre avec des enfants de trois à cinq ans. Je relis Piaget, Wallon, Winnicott avec le sentiment de comprendre ce que j'étudiais plus tôt et je décide d'écrire un texte pour les plus petits.

Ce sera Une lune entre deux maisons ${ }^{4}$. L'écriture de ce texte, la création du spectacle, la fréquentation des petits durant les représentations et les rencontres après le spectacle, l'étude de leurs dessins ont été ma première vraie école d'écriture. J'ai reçu comme Jacotot, «le maître ignorant» de Jacques Rancière, la conviction que je devais apprendre des enfants. L'écriture de Une lune entre deux maisons m'a appris que je devais arrêter de regarder et d'expliquer le monde du haut de ma taille d'adulte, que je n'avais rien à faire "comprendre" aux enfants, que je devais m'asseoir par terre avec eux, regarder le monde les yeux à la hauteur des leurs et les écouter parler du monde dans leurs mots, leurs phrases, leurs hésitations et leurs certitudes, leurs rêves et leurs peurs.

Après Une lune entre deux maisons, je n'ai plus jamais écrit innocemment. J'ai écrit, en sachant que j'écrivais pour enfants et avec la conscience intime que j'étais auteure, femme, mère, adulte, être humain et que je devais inventer pour chacun de mes textes un équilibre instable et précaire entre l'auteure que j'étais et un public spécifique, captif, toujours nouveau, multiple. Qui pourrait nier qu'entre un enfant de trois ans et un de douze ans, il y a une distance aussi grande qu'entre l'enfant et l'adulte, que deux enfants de trois ans ont des expériences qui en font déjà des êtres humains uniques?

4. S. Lebeau, Une lune entre deux maisons, Québec/Amérique, I980; Montreuil, Éditions Théâtrales Jeunesse, 2006. 


\section{La commande impossible}

Il aura fallu mon adaptation' de Quand j'avais cinq ans j'm'ai tué de Howard Buten pour théoriser ce que j'appelle la commande contradictoire (celle de l'intérieur et celle de l'extérieur) et comprendre le pari impossible d'obéir à deux dynamiques aussi fondamentalement opposées : celle d'adopter un point de vue autre et celle d'être totalement en accord avec ce que j'écrivais dans le dire et dans la manière de dire. Avec Quand j'avais cinq ans j'm'ai tué, j'ai été attaquée violemment et de plein fouet... Mais ce texte, je ne l'avais pas écrit mais choisi et je pouvais défendre mon choix : le roman décrit une société et ses tabous à travers le regard d'un enfant de huit ans et s'aventure dans des zones émotives que, toute seule, je n'aurais peut-être pas explorées.

Le jour de la première, l'émotion est à couper au couteau. Les enfants sont attentifs, silencieux. Les adultes, effrayés, désemparés autant par le propos que par le ton "grave» du spectacle. L'expérience de Quand javais cinq ans, j'm'ai tué m'a permis de comprendre le paradoxe fondamental du théâtre pour enfants qui fait de cette pratique sous haute surveillance un exercice de haute voltige pour les créateurs qui s'écartent des voies permises : fantaisie et didactisme. J'ai appris :

- que la compréhension des enfants est subtile, globale, profonde, qu'elle va plus loin que ce que l'on peut imaginer, prévoir, définir;

- que les enfants sont disponibles, sans préjugés quant aux propos, ouverts aux formes les plus contemporaines;

- que les adultes ont des idées précises sur ce qui peut être ou ne peut pas être "dit aux enfants», que ces idées sont totalement subjectives, basées sur des souvenirs d'enfance, celle qu'ils ont eue ou n'ont pas eue et surtout teintées de la relation de subordination didactique où l'adulte est celui qui sait et l'enfant, celui qui apprend;

- que s'il y a des limites à ce que l'on peut leur dire ou montrer, ces limites ne viennent jamais des enfants mais des adultes qui les entourent;

- que l'enfance, même la plus choyée, la plus lumineuse, n'est jamais complètement idyllique : il suffit de penser à la colère d'un tout-petit...;

- que les enfants vivent dans le même monde que nous, curieux, ouverts aux réalités qu'ils devinent, soupçonnent et... le silence sur les problématiques complexes est plus douloureux que la discussion soulevée par un spectacle, un film, un livre.

5. S. Lebeau, Gil, texte inédit, 1987. 


\section{La métaphore fondatrice}

L'expérience de Quand j'avais cinq ans j'miai tué m'a permis de nommer la difficulté à écrire pour le jeune public : réconcilier l'inconciliable. Recueillir et connaitre des points de vue d'enfants sur des questions existentielles qui me concernent à un moment donné de ma vie sans trahir : ne pas trahir les confidences des enfants et leurs mots, ne pas me trahir moi-même dans ma manière de dire avec un souffle, un rythme, un vocabulaire, des images et un imaginaire... L'alchimie est délicate et cela à toutes les étapes de l'écriture.

La pulsion, l'impulsion qui déclenchent les premières questions peuvent être internes (des questions obsédantes), ou externes : le choc d'un livre, d'un film, d'un documentaire que je n'arrive pas à oublier, qui s'installe à mon insu comme matière d'écriture. C'est alors que surgit en arrière-plan la question du ou des publics qui se pose de manière complètement différente pour chacun des projets. Je n’ai jamais réussi à dégager suffisamment de constantes pour reproduire une approche, modéliser une manière de faire. Je suis déjà dans la création. La relation est à inventer chaque fois à partir d'un désir, d'une intuition, d'un flou impalpable et unique, d'une recherche dont le point de départ est un point d'interrogation et le point d'arrivée de multiples points d'interrogation. Chercher... imaginer... inventer des méthodes, des moyens, des questions, des plans de travail, des objectifs, des défis avec des mots, des images, les miens et ceux des enfants pour m'approcher d'une réalité, d'une situation, d'un vécu.

C'est ainsi que j'ai vécu les périodes d'animation faites avec les enfants pour chacun de mes textes. J'ai écrit Salvador ${ }^{6}$ à la suite de deux voyages en Argentine et au Pérou où j'ai connu les enfants des rues. Je ne sentais pas de difficulté particulière à partager l'expérience avec les enfants du Nord quand je suis allée rencontrer les enfants près de chez moi pour les entendre parler de réalités qu'ils ne vivaient pas. Leurs commentaires ont été des avertissements sérieux : je devais éviter le misérabilisme et appeler à l'espoir. J'ai choisi un destin heureux, ce que je n'aurais peut-être pas fait si j'avais écrit pour adultes. Je ne me trahissais pas pour autant : j'avais moi-même besoin de le faire exister, ce destin.

Le déclencheur de $\mathrm{L}^{\prime} O$ grelet ${ }^{7}$ était une question existentielle, la pièce questionne les pulsions du bien et du mal. Petit Pierre ${ }^{8}$ posait la question

6. S. Lebeau, Salvador, L'Enfant, la montagne et la mangue, Montréal, VLB Éditeur, I994; Montreuil, Éditions Théâtrales Jeunesse, 2002.

7. S. Lebeau, L'Ogrelet, Montréal, Lanctôt Éditeur, I997; Montreuil, Éditions Théâtrales Jeunesse, 2003.

8. S. Lebeau, Petit Pierre, Montréal, Lanctôt Éditeur, 2002 ; Montreuil, Éditions Théâtrales Jeunesse, 2006. 
universellement connue des enfants et des adultes de ne pas être conforme. Souliers de sable réconcilie ma haine des dispositifs de sécurité avec le besoin inné qu'ont les petits d'explorer. Gretel et Hansel ${ }^{\text {to }}$ abordait la jalousie fraternelle que nous passons notre vie à actualiser et réactualiser.

Je termine cette revue tronquée en posant les balises du travail d'animation avec les enfants avant d'aborder l'écriture du texte Le bruit des os qui craquent ${ }^{I I}$. J'ai demandé crûment : "Est-ce qu'on a le droit de parler de réalités aussi dures à des enfants qui ne les connaissent pas?» Leurs réponses sans équivoque m’ont permis d'écrire ce texte qui continue de poser des questions : pourquoi ce texte écrit pour les enfants se retrouve-t-il régulièrement dans les programmations adultes? Autre question, plus bouleversante encore : pourquoi Le bruit des os qui craquent provoque-t-il deux lectures divergentes, se révélant une histoire d'espoir pour les enfants et une histoire désespérante pour les adultes?

J'écris avec la certitude d'un décalage fondamental, d'une distance à première vue irréconciliable entre l'artiste et le spectateur. Comme je le sais inévitable, j'ai décidé d'en fouiller les interstices, les possibles et les impossibles, les pièges, les fausses pistes et il est devenu un passionnant défi. Il m’oblige à rester près des enfants durant le processus d'écriture, à définir ce que moi, femme, adulte, citoyenne, je veux partager avec le public et à être intimement attentive aux forces conscientes et inconscientes de l'écriture pour que s'opère la perte de contrôle du geste artistique, contrôle que les adultes se sentent obligés de garder dans leur comportement avec les enfants... Comment cette perte de contrôle, essentielle à la création, s'est-elle opérée dans l'écriture de chacun de mes textes?

Il y a vingt ans à peine, j'ai senti qu'il y avait un moment précis où l'écriture s'enclenchait. Il y a dix ans à peine, j'ai nommé de manière intuitive et empirique le moment précis où se met en place la mécanique de l'écriture qui se détache complètement des étapes qui l'ont précédée : j'ai appelé ce moment métaphore fondatrice. La métaphore est l'image sans outil de comparaison qui associe un terme à un autre, ce deuxième terme appartenant à un champ lexical différent afin de traduire une pensée plus riche et plus complexe que celle du vocabulaire descriptif concret. Ce serait une figure de rhétorique qui dit entre les lignes, entre les lettres et qui, surtout, dit plus que la somme de ses composantes. Le mot me donne le sentiment intangible mais délicieux d'une étonnante clarté dans le fouillis qu'imposait la digestion silencieuse qui suivait les animations

9. S. Lebeau, Souliers de sable, Montréal, Leméac, 2006; Montreuil, Éditions Théâtrales Jeunesse, 2007.

Io. S. Lebeau, Hansel et Gretel, Montreuil, Éditions Théâtrales Jeunesse, 2014.

II. S. Lebeau, Le bruit des os qui craquent, Montréal, Leméac, 2009; Montreuil, Éditions Théâtrales Jeunesse, 2008. 
avec les enfants. Je l'ai appelée fondatrice parce qu'elle me permettait d'articuler et de faire vivre dans une histoire, une situation, un personnage les paradoxes, les contradictions, les irréconciliables que la recherche avait fait surgir : l'idée du petit de l'ogre qui ouvre sur tous les possibles, la structure double qui permet l'émotion en direct et la mise en contexte, l'alternance entre récit et action, entre conte et modernité. Tous mes textes s'articulent autour d'une métaphore.

La métaphore fondatrice est devenue le pivot de mon écriture marquant systématiquement le passage involontaire, parfois inconscient, mais définitif entre la fin de la recherche (commande de l'extérieur) et l'écriture (commande de l'intérieur) qui suppose perte de contrôle et abandon aux détours de l'inconscient. L'écriture s'impose, se nourrit d'elle-même et des sens qu'elle fait surgir, des mots, des images, au rythme des émotions. La métaphore fondatrice a le pouvoir de faire taire les milliers d'hypothèses que la recherche ou le désir conscient ont fait surgir pendant la gestation, le pouvoir de les englober toutes, d'en choisir une qui les raconte toutes. Elle est féconde et, si elle est chemin pour l'auteur, elle ouvre des voies de traverse pour que chaque spectateur retrouve son intimité.

La magistrale démonstration de l'évolution historique et culturelle du concept de métaphore de Paul Ricœur ${ }^{12}$ m'a permis de mettre des mots et des concepts sur des intuitions. Déjà, chez Aristote, la métaphore est définie comme mouvement qui unit une paire de termes. Il faut deux idées pour une métaphore et le plus souvent l'idée de transgression catégoriale enrichit l'écart entre les deux termes. Cette transgression n'est intéressante que lorsqu'elle produit du sens, fonction que j'attribue à la métaphore fondatrice : créer un sens qui n'existait pas, troisième terme à partir de termes contradictoires, de visions ou de points de vue souvent irréconciliables. Si, comme Aristote le disait, une des fonctions de la métaphore était de combler une lacune sémantique, j'ai toujours cru que la métaphore était ce pont entre le monde des enfants et celui des adultes que l'on oppose de manière radicale et sans justification. Il suffit de penser aux contes d'Andersen, "Le vilain petit canard», "La petite marchande d'allumettes»...

Max Black, toujours cité par Ricœur, va plus loin, affirmant que la métaphore défait un ordre pour en refaire un autre et qu'on est alors dans la logique de la découverte. Max Black permet de faire le lien entre concept épistémologique et concept poétique quand il avance que la métaphore appartient à l'ordre du discours et de l'œuvre puisque la stratégie du langage propre à la poésie consiste bien dans la constitution d'un sens qui intercepte la référence et, à la limite, abolit la réalité. Enfin, plusieurs des auteurs analysés par Ricœur

I2. P. Ricœur, La Métaphore vive, Paris, Seuil, 1997. 
parlent abondamment de la tension créatrice qui jaillit de la collision sémantique de deux termes, deux visions, deux points de vue : la métaphore serait un court-circuit de réalités polarisées saisies dans une troisième dimension qui les réconcilie en leur gardant leur caractère contradictoire. Selon Black, la métaphore est au langage poétique ce qu'est le modèle au langage scientifique, dans la relation au réel. On sait que dans le langage scientifique, le modèle est un instrument heuristique qui sert à briser une interprétation non adéquate et à ouvrir la voie à une nouvelle interprétation.

Il me reste à analyser chacun de mes textes pour voir comment s'est déployée la métaphore fondatrice, quelles contradictions elle devait prendre en compte, comment elle travaillait les personnages, les situations, se nourrissait d'archétypes, de contes, de mythologies, portée par des réseaux souterrains de sens et d'images que nous recevons à la naissance, tels dans une culture particulière et tels de manière universelle. Ricœur explique bien que la métaphore est portée par un réseau. Il ne s'agit plus de simples connexions nouvelles mais d'une philosophie de l'imagination. Il rejoint en cela Bachelard et Durand, des maitres qui m’ont permis de nourrir les silences de l'écriture en fouillant au plus loin, à l'intérieur, pour établir des correspondances inconscientes entre le reçu des enfants et mon vouloir dire. Il me faudra poser la question philosophique de la vérité métaphorique chère à Ricœur. Quelle serait cette vérité métaphorique? J'ose une hypothèse qui ne concerne que mon travail et moi-même, mais je veux la vérifier : elle serait peut-être cette tension fertile qui fait naître l'étrange, l'inconnu, le neuf... et ce terrain neutre, terrain commun plutôt, où adultes et enfants sont des humains...

\section{Le point de vue des neurosciences}

Mon effort pour réduire les paradoxes inhérents à l'écriture pour le jeune public m'a amenée au concept d'empathie auquel les neurosciences ont donné une extension (entre autres dans le processus de création) et une crédibilité scientifique (l'IRM a permis des avancées déterminantes dans la connaissance du cerveau et des processus cognitifs). Je fais l'hypothèse que le concept d'empathie m'aidera à comprendre pourquoi certains de mes textes ont heurté si fort les adultes alors qu'ils touchaient précisément les jeunes publics, pourquoi je me suis retrouvée si souvent au cœur des débats sur ce qui peut ou non se dire aux enfants, pourquoi mon écriture s'est laissé déranger ou nourrir de questionnements qui me ramenaient toujours à l'essentialité de l'humain, aux doutes existentiels qui nous troublent de la naissance à la mort, loin des images consensuelles d'une enfance légère, même dans son imaginaire. Enfin, j'ai reconnu dans le concept d'empathie 
ma méthode de travail : en 1988 à propos d'Une lune entre deux maisons, qui marque un tournant dans ma manière d'écrire pour enfants, j'affirmais qu' «Écrire pour les enfants est davantage une façon de regarder le monde que la nécessité d'en inventer un différent et merveilleux, plus gai, plus coloré, plus souriant ${ }^{13}{ }^{13}$. Il s'agit bien d'empathie au sens où l'entend Alain Berthoz:

Le secret de l'empathie ne se trouve pas seulement dans les neurones miroirs. Il ne réside pas non plus seulement dans la capacité de simuler mentalement les actions de l'autre ou d'en éprouver les émotions. Il exige cette capacité de changer de point de vue tout en gardant le sentiment de soi ${ }^{14}$.

Alain Berthoz ajoute que si le changement de point de vue est essentiel... Il ne suffit pas. Il faut également un "changement de point de sentir [...] en intégrant dans le flux de son vécu l'expérience de l'autre ${ }^{15} »$.

J'aimerais analyser mes préparations d'animation avec les enfants pour chacun des textes écrits depuis trente-cinq ans : documents amassés, processus d'animation, de consultation, moments clefs où je rencontrais les enfants avec des questions précises, influence des conclusions que je tirais de ces rencontres sur les métaphores fondatrices, sur l'écriture elle-même. J'aimerais évaluer qualitativement et quantitativement les munitions et argumentaires que les consultations auprès des enfants me donnaient pour affronter les adultes et répondre aux objections que l'on faisait sur la pertinence d'un contenu, d'une forme. Je ferais à rebours trente-cinq ans de création à la lumière du concept éclairant qu'est l'empathie qui suppose la capacité d'adopter le point de vue de l'autre sans jamais oublier son propre point de vue. Je citerai Alain Berthoz en forme de conclusion :

Il me semble que nous avons devant nous un curieux processus dynamique d'interaction vécue qui exige simultanément d'être soi et un autre, de se vivre soi-même et en même temps d'échapper à ce point de vue égocentré pour adopter un point de vue hétérocentrér ${ }^{16}$.

13. S. Lebeau, dans F. Villaume (dir.), Le choix de Suzanne Lebeau, Montreuil, Éditions Théâtrales, 2013, p. 79.

I4. A. Berthoz, G. Jorland (dir.), L'empathie, Paris, Odile Jacob, 2004, p. 263.

I5. Ibid., p. 266.

I6. Ibid., p. 254. 